\title{
Aspectos florísticos de 13 hectares da área de Cachoeira Porteira-PA ${ }^{1}$.
}

\author{
Diógenes de Andrade LIMA FILHO ${ }^{2}$, Juan REVILLA ${ }^{2}$, Iêda L. do AMARAL ${ }^{2}$, Francisca Dionizia de A. MATOS ${ }^{2}$, \\ Luiz de Souza COÊLHO ${ }^{2}$, José Ferreira RAMOS², Gláucio Belém da SILVA², José de Oliveira GUEDES.
}

\section{RESUMO}

Este trabalho descreve a composição florística na área do reservatório da futura Hidrelétrica de Cachoeira Porteira - PA (localizada na margem esquerda do rio Trombetas), e a caracterização da vegetação. São apresentados dados sobre a abundância, dominância, freqüência e os Índices de Valor de Importância das espécies (IVIE) e o Índice de Valor de Importância das Famílias (IVIF) e a análise da estrutura horizontal da floresta. Os estudos desenvolvidos neste trabalho mostram as espécies e famílias mais importantes da área com relação a sua influência, na definição do perfil estrutural da floresta, além da identificação dos diferentes tipos de vegetação. Os 13 hectares de floresta inventariados sustentam 4.583 indivíduos, abrangendo árvores, palmeiras e cipós com DAP $>10 \mathrm{~cm}$, distribuídos em 359 espécies, 217 gêneros e 55 famílias (três medidas de importância ecológica; abundância, dominância e freqüência, expressas como três porcentagens, foram somadas para obter um Índice de Valor de Importância (IVIE) das espécies). As duas espécies com os maiores IVIE, em toda a área estudada pesquisada, foram Eschweilera coriacea (DC) S. A. Mori, com 15,24\% e Micropholis guyanensis (A . DC.) Pierre com 10,87\%. As famílias que apresentaram os maiores Índices de Valor de Importância (IVIF) nos 13 hectares, foram Caesalpiniaceae (31,45\%) e Sapotaceae (30,34\%).

\section{PALAVRAS-CHAVE}

Amazônia, Tipologia florestal, Inventário florístico

\section{Floristic composition of 13 bectars in Cachoeira Porteira, Pará state}

\begin{abstract}
This work describes the floristic composition in the future Cachoeira Porteira UHE-PA water reservoir area (located on the left margin of the Trombetas river), and the characterization of the vegetation. Data on abundance, dominance, frequency, Species Importance Value Indexes (IVIE), Family Importance Value Index (IVIF), and forest borizontal structure analysis, are presented in the present study. The studies developed in this work show the area's most important species and families, according to their influence on the forest structural profile definition, in addition to the identification of vegetation different types. The 13 surveyed forest hectares support 4.583 individuals, comprising trees, palms and lianas with DAP $>10 \mathrm{~cm}$, distributed in 359 species, 217 genera and 55 families (Three measures of ecological importance were totaled to give an Importance Value Index (IVIE) of the species). The two species with the bighest IVIE, in all surveyed area, were Eschweilera coriacea (DC) S. A. Mori, with 15,24\% and Micropholis guyanensis (A. DC) Pierre, with 10,87\%. Families presenting the bighest Importance Value Indexes (IVIF) in the 13 hectares, were Caealpiniaceae (31,45\%) and Sapotaceae $(30,34 \%)$.
\end{abstract}

\section{KEY WORDS}

Amazônia, forest tipology, forest inventory

\section{INTRODUÇÃO}

A região amazônica vem sofrendo interferência humana inadequada nos últimos 20 anos, exigindo da sociedade uma perspectiva de aproveitamento sócio-econômico mais elaborada e consistente, no aspecto do conhecimento da sua cobertura vegetal. No entanto, a Amazônia é uma região bastante heterogênea no que diz respeito a sua composição florística, evidenciando a complexidade dos grupos vegetais que a compõem.

${ }^{1}$ Financiado pelo convênio ELETRONORTE/INPA/MCT.

${ }^{2}$ Coordenação de Pesquisas em Botânica Instituto Nacional de Pesquisas da Amazônia, INPA/CPBO. Alameda Cosme Ferreira 1756, Aleixo, Manaus - Am. CEP. 69083-000. 
Montoya Maquin \& Matos (1967) descrevem que a vegetação da Amazônia pode ser estudada, mediante vários parâmetros, visando a sua classificação. Rizzini (1963) afirma que a organização das unidades da estrutura fisionômica e florística da região amazônica é fundamental para o conhecimento científico. Alencar (1986) descreve a vegetação através dos parâmetros estruturais como abundância, dominância e freqüência.

Atualmente há inúmeras informações sobre diversidade florística, Oliveira (2000) apresenta um histórico, documentando os inventários realizados na Amazônia. Assim, esses autores visam classificar a vegetação da região amazônica utilizando vários métodos para estudar a estrutura da vegetação. Mas, poucos destes autores levaram em consideração o relacionamento destas com o meio ambiente. Com isso, a preocupação do conhecimento e reconhecimento de algumas áreas da Amazônia Brasileira tem sido um desafio para a ciência florestal. Na futura UHE de Cachoeira Porteira - PA, procurouse determinar alguns parâmetros para conhecimento das espécies vegetais que compõe aquela região.

Neste trabalho, realizou-se levantamento florístico de treze (13) unidades amostrais $(10 \times 1000 \mathrm{~m})$ em área escolhida para esse fim.

\section{MATERIAL E MÉTODOS}

\section{Caracterização da área de estudo}

Os levantamentos foram realizados na área prevista para o futuro reservatório da UHE de Cachoeira Porteira - PA, localizada no município de Oriximiná-PA como mostra a Figura 1, limitada pelas seguintes coordenadas: latitude $0^{\circ}$ $30^{\prime} \mathrm{S}$ à $1^{\circ} 03^{\prime} \mathrm{S}$ e longitude $56^{\circ} 49^{\prime} \mathrm{W}$ à $57^{\circ} 37^{\prime} \mathrm{W}$. A área mostra um grau bastante heterogêneo em relação à sua constituição florística.

\section{Clima}

Segundo a classificação de Köppen, a região estudada apresenta o tipo climático Awi, que tem como característica principal uma estiagem de três meses geralmente nos meses de outubro, novembro e dezembro. A região tem temperatura média anual de $26,5^{\circ} \mathrm{C}$, pluviosidade um pouco reduzida, ou seja, $2.000 \mathrm{~mm}$ a $2.500 \mathrm{~mm}$ anuais aproximadamente.

\section{Solos}

De acordo com Revilla et al. (1986), em Cachoeira Porteira o solo varia desde arenoso até granítico, argiloso, pedregoso, hidromórfico e xeromórfico, ficando mais complexo ainda pelos relevos apresentados na área, onde em muitos locais essa região supera os $100 \mathrm{~m}$ de altura. Mas, no geral nesta área predomina o solo podzólico vermelhoamarelo (PV).

\section{Vegetação}

Predomina a mata de terra firme, a qual é influenciada pelo relevo acidentado de algumas áreas, além de outras planas e mistas em algumas zonas. As árvores se apresentam com uma altura média aproximadamente de $25 \mathrm{~m}$ e com diâmetro à altura do peito (DAP) em média, de $30 \mathrm{~cm}$.

\section{Procedimentos}

Foi inicialmente utilizada a fotointerpretação das fotografias aéreas na escala 1:40.000 com mapas de 1:100.000, e o fotoíndice, empregando a metodologia clássica de fotointerpretação.

Após a constatação dos diferentes tipos de vegetação (sete tipologias vegetais) a partir do mapa, foram préescolhidos os pontos estratégicos de estudo. Logo depois, as unidades de amostra foram alocadas mediante a análise do mosaico fotográfico da imagem da área.

O modelo proposto por Lamprecht (1964), Finol (1971) e utilizado por Longhi (1980), Revilla et al. (1981), Matos \& Amaral (1999) e Lima Filho et al. (2001), o qual consiste em uma faixa de $10 \times 1000 \mathrm{~m}$ ( $1 \mathrm{ha})$, dividida em parcelas de $10 \times 50 \mathrm{~m}$, serviu de base para a forma e o tamanho das unidades amostrais estabelecidas.

Empregou-se o método misto (sistemático-aleatório), ou seja, sorteando a primeira unidade de amostra, e as demais seguiram em função do posicionamento dos pontos cardeais, e alocando-as aleatoriamente nos diferentes tipos de vegetação (Lima Filho et al. 2001). Uma vez demarcadas, as parcelas contíguas, procedeu-se à coleta de informações biométricas (DAP, altura do fuste e altura total) de todas as árvores que possuíam DAP $\geqslant 10 \mathrm{~cm}$, incluindo cipós e palmeiras. Em seguida, procedeu-se à coleta botânica, cujos exemplares foram submetidos à secagem por 48 horas em estufas a $60{ }^{\circ} \mathrm{C}$ e posterior identificação do material no herbário do INPA, cuja classificação obedece ao sistema de Cronquist (1981).

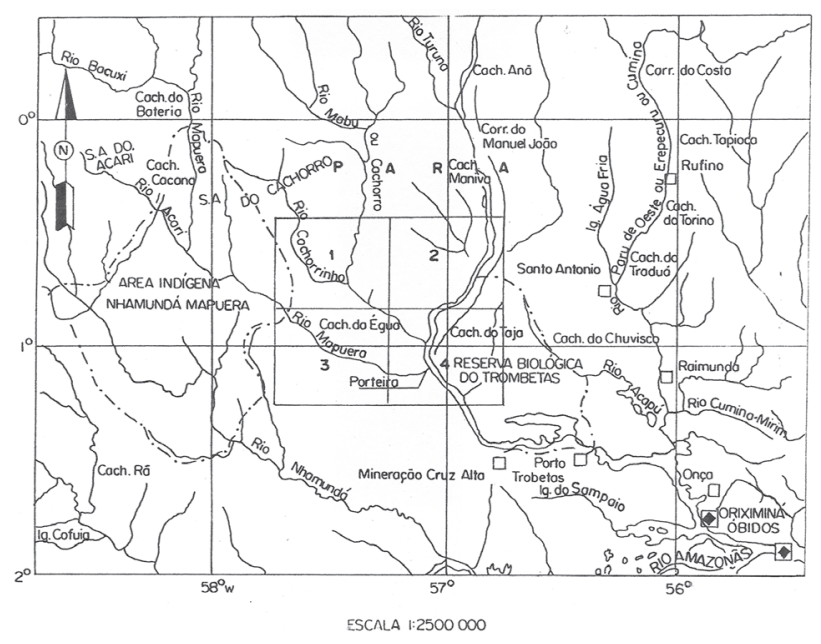

Figura 1 - Localização da área de estudo em Cachoeira Porteira - PA 


\section{ACTA \\ AMAZONICA}

A composição florística foi analisada a partir dos resultados obtidos dos números de indivíduos, espécies, gêneros e famílias, que ocorreram na área de estudo.

A estrutura horizontal foi analisada com o uso de critérios que indicam a ocupação do solo pela espécie, no sentido horizontal. Os parâmetros estudados foram: abundância absoluta (Lamprecht, 1962; 1964), que é o número de plantas de cada espécie, em relação à unidade de área e a abundância relativa que é definida pela percentagem da abundância absoluta na composição florística do povoamento (Souza,1973; Veiga, 1977). A dominância absoluta (Cain et al., 1956; Lamprecht, 1964; Finol, 1971; Hosokawa, 1981; Jardim, 1985; Conceição, 1990) é obtida pela soma das secções transversais dos indivíduos de uma espécie por unidade de área, enquanto que a dominância relativa é calculada pela percentagem do total das dominâncias absolutas (área basal/ha), e corresponde à participação em percentagem de cada espécie na expansão horizontal total. A freqüência absoluta (Förster, 1973) representa o número de subparcelas em que ocorre uma espécie, dividido pelo número total de subparcelas em que foi dividida a amostra e a freqüência relativa é a soma das freqüências absolutas das parcelas. O Índice de Valor de Importância das espécies (IVIE) é a soma da abundância, dominância e freqüência relativas em uma única expressão, desses três dados estruturais, segundo Cain et al. (1956) e Cain \& Castro (1959). Por último, o Índice de Valor de Importância das Famílias (IVIF) que é a combinação entre o IVIE das espécies pertencentes à mesma família. Mori et al. (1983), propõem que este índice seja obtido pelo somatório do cálculo dos parâmetros da diversidade, densidade e dominância relativas, o que foi adotado no presente trabalho.

\section{RESULTADOS E DISCUSSÃO}

\section{Tipologias florestais}

Os resultados de campo mostraram as principais comunidades observadas, as quais foram classificadas de acordo com Revilla et a.l (1986), onde se tem:

\section{a- Floresta densa de terra firme sobre relevo plano ou mata de terra firme}

Caracteriza-se por apresentar vegetação uniforme no seu dossel, onde se encontram árvores com troncos moderadamente esparsos, retos com início das ramificações sempre no alto do fuste, copas amplas de formas globosas e de guarda-chuva. O dossel apresenta poucas epífitas e cipós, mantendo uma variação na altura com média no intervalo de 25 a 30m, evidenciando de maneira clara espécies emergentes. O solo apresenta-se em alguns pontos com afloramentos das rochas e mal drenado. A camada de serapilheira é moderada, oscilando em espessura de 20 a $30 \mathrm{~cm}$.
$\mathrm{O}$ estrato inferior (até $5 \mathrm{~m}$ ) normalmente esparso ou denso, quando existem clareiras no dossel, está constituído pela regeneração natural, onde se observou também a presença de pequenas palmeiras como "ubim" (Geonoma sp.), (Lepidocaryum tenue Mart.), (Scheelea sp.) entre outras.

O estrato médio (5-15m) não difere muito do anterior, mas apresenta baixa diversidade de espécies, dentre as quais destacam-se "bacaba" (Oenocarpus bacaba Mart.), "tucumarana" (Astrocaryum sp.), "gitó" (Guarea carinata Ducke) e "ingarana" (Inga sp.).

Já no estrato superior (25-30m), destacam-se "matá-matá branco" (Eschweilera sp.), "angelim - rajado" (Zygia racemosa Barneby \& J. W. Grimes), "tachi” (Tachigalia sp.) e "piquiá" (Caryocar villosum Aubl.).

\section{b- Floresta densa de terra firme sobre relevo dissecado}

Caracterizada por apresentar relevo acentuado até formar serras isoladas (bacia do rio Trombetas) ou serras contínuas (rio Cachorro), o substrato destas serras quase sempre é rochoso ou em seixos grossos, porém em algumas áreas pode-se observar rochas compridas expostas por vários metros.

A cobertura vegetal desta comunidade é bastante heterogênea, assim temos, nos platôs das serras, morros com uma vegetação uniforme e contínua, onde se observa a vegetação herbácea tipo rupreste com espécies das famílias Bromeliaceae, Polypodiaceae, Selaginellaceae e Piperaceae. Entretanto, nas encostas pouco pronunciadas podemos observar uma cobertura vegetal densa, porém com árvores altas e esparsas que caracterizam a mata de cipó (Pires, 1973) conhecida assim pelo papel que desempenham os cipós que cobrem os afloramentos rochosos e sítios que outras espécies não conseguiram colonizar.

\section{c- Floresta densa de terra firme sobre relevo ondulado}

Caracteriza-se pela presença de colinas pouco acentuadas, tem aparência de ondulações contínuas e o relevo reflete-se claramente no dossel, ou seja, manifestase também em ondulações das árvores que compõem a vegetação do estrato superior. Porém, a estrutura difere pouco da comunidade anterior, mas existe um número maior de pequenas palmeiras no sub-bosque. O acúmulo de serapilheira é maior nas encostas das colinas e menor nos platôs, entretanto a drenagem é melhor que a comunidade anterior.

O estoque de fitomassa é menor que a comunidade anterior. A explicação está relacionada ao maior espaçamento da distribuição das árvores, principalmente nos declives e zonas mais baixas, onde o dossel é mais aberto. 


\section{ACTA AMAZONICA}

ASPECTOS FLORISTICOS DE 13 HECTARES

DA ÁREA DE CACHOEIRA PORTEIRA-PA

\section{d - Floresta mista ciliar estacionalmente inundável ou mata de igapó}

Caracteriza-se pela influência anual do nível da água, situando-se sempre nas margens dos rios. Este fato, determina a presença de espécies com adaptações que permitam suportar até 8 meses de submersão e/ou alagação. A terminologia desta é diversa destacando-se a floresta de igapó (Revilla et al., 1981) e mata de várzea (Rodrigues, 1961), o fato é que essas espécies que vivem nestas condições são diferentes de qualquer outra comunidade.

A estrutura desta floresta é diversa, pois existem áreas de mata alta (até $30 \mathrm{~m}$ ) e próxima a estas, mata baixa, constituída por arbustos e arvoretas. Observouse que a mistura destas comunidades vegetais se dá principalmente por causa da posição dos meandros do rio, dando origem às praias de um lado e formando barrancos no outro. Assim, logo após a floresta marginal alta tem-se grandes áreas inundáveis, e que apresentam uma certa uniformidade parecida à mata de baixio, porém tem uma flora característica na qual destacamse as taperebá " (Spondias lutea Lind.), " cajurana " (Simaba guianensis Aubl.), " munguba " (Pseudobombax munguba Mart.), " macucu - de - igapó " (Aldina latifolia Spruce) e outras espécies arbóreas que geralmente deixam espaços, como exemplo temos as touceiras de " jauari " (Astrocaryum jauari Mart.) que se destacam nestas matas. O número de cipós é importante para a fisionomia desta mata, onde encontram-se as espécies Maripa sp., Salacea sp., Memora sp., Arrabidea sp. e Hippocratea sp., que destacaram-se na área de estudo. É bom salientar que nesta comunidade, a maioria das espécies têm uma grande produção de frutos e sementes que alimentam as populações de peixes.

\section{e - Floresta mista descontínua de terra firme e/ ou mata de campina e campinarana}

Ocorre sobre solo arenoso e apresenta caracteres de escleromorfismo principalmente nas áreas bem drenadas.

A denominação de campina e campinarana da Amazônia Central (Lisboa, 1975) difere das campinas do sul do país. A campina apresenta vegetação aberta como ilhas, deixando o solo arenoso exposto em algumas áreas, as alturas são variáveis sendo de poucos centímetros até $5 \mathrm{~m}$, as ilhas de mata que são características desta comunidade, apresentamse globosas com troncos e galhos tortuosos, escleromorficos e raquíticos. O sub-bosque sempre com grande quantidade de folhagens e galhos secos, formando grandes tufos, que servem de substrato onde vivem algumas espécies de orquídeas e bromélias. As espécies arbóreas mais freqüentes são " umiri " (Humiria balsamifera Aubl.), " pimenta-delontra " (Ouratea spruceana Engl.), " tento " (Ormosia costulata Miq.).

A campinarana é considerada como um estágio mais desenvolvido da campina, e caracteriza-se por ter sua estrutura mais uniforme e contínua, porém ainda é uma floresta baixa (no máximo $15 \mathrm{~m}$ de altura aproximadamente), com troncos e galhos mais esparsos e com diâmetro de $20-30 \mathrm{~cm}$ de DAP. As espécies que mais se destacaram foram " muirajibóia - preta " (Bocoa viridiflora Ducke), “ pau - jacaré “ (Laetia procera Poepp.), " muirapiranga " (Eperua glabriflora Ducke ), " maparajuba " (Manilkara bidentata A .DC. ), " pente- de - macaco “ (Apeiba echinata Gaertn.).

\section{f- Mata de bambu}

Ocorre geralmente em terrenos ondulados com formações de platôs e pequenas serras. O solo em geral é argiloso e bem drenado, ocorrendo modificações nas zonas baixas onde sempre existe igarapé. A camada de serapilheira é de aproximadamente $20-40 \mathrm{~cm}$, isto em zona alta, já na zona baixa a característica física do ambiente modifica um pouco quanto à vegetação, porque não há predominância de bambu, mas sim de uma vegetação típica de terra firme, porém sem haver uma exuberância nas árvores. Observouse que o solo modifica-se, tornando-se argilo - arenoso e o acúmulo de serapilheira tende a ser menor. As duas espécies que obtiveram maior destaque foram " piquiá " (Caryocar villosum Aubl.) e a "envireira " (Xylopia sp.).

\section{g- Mata secundária ou capoeira}

Após a influência antrópica, a mata nativa passa a ser considerada como " capoeira " apresentando o pobre estoque de biomassa com a vegetação descontínua em alguns setores (moitas).

A composição florística desta comunidade está ligada à idade da própria capoeira e das interferências humanas que estas áreas sofreram. Assim, observaram-se áreas onde existem dominância de gramíneas como o " rabo - de raposa " (Andropogon bicornis Benth.), "capim - roxo" (Paspalum conjugatum Berg.), "capim - tiririca" (Scleria platensis Lindl.) até espécies do tipo pioneira "imbaúba" (Cecropia leucocoma Miq.), "Goiaba - de - anta" (Bellucia grossularioides Trian.), "buxixu - anil" (Miconia chrysophylla Rich.).

Com isso, pode-se dizer que a vegetação encontrada tem uma relação muito estreita com o tipo de solo, situação de drenagem e relevo, tornando a tipologia florestal da área muito diversificada. Podem ser observados alguns tipos de vegetação, pela maneira geral das descrições tradicionais, porém olhando-se em detalhes podem ser consideradas como mosaicos, e estes, no momento de definir seu acoplamento verifica-se que é muito mais complexo pela existência marcante do relevo, quando este varia acima de $10 \mathrm{~m}$. Desta maneira, é difícil conciliar uma ou mais tipologias, sobre tudo, realizar extrapolação da área basal e volume, sendo ainda mais difícil a mudança florística. 


\section{ACTA AMAZONICA}

Tabela 1 - Distribuição do número de indivíduos (DAP $\geqslant 10 \mathrm{~cm})$, gêneros e espécies por família na área de Cachoeira Porteira / PA.

\begin{tabular}{|c|c|c|c|}
\hline FAMÍLIA & ESPÉCIES & GÊNEROS & INDIVÍDUOS \\
\hline Anacardiaceae & 9 & 5 & 55 \\
\hline Annonaceae & 14 & 9 & 154 \\
\hline Apocynaceae & 18 & 9 & 70 \\
\hline Araliaceae & 1 & 1 & 4 \\
\hline Arecaceae & 9 & 8 & 165 \\
\hline Bignoniaceae & 4 & 2 & 18 \\
\hline Bombacaceae & 7 & 4 & 20 \\
\hline Boraginaceae & 2 & 1 & 17 \\
\hline Burseraceae & 6 & 3 & 461 \\
\hline Caesalpiniaceae & 36 & 20 & 440 \\
\hline Caryocaraceae & 4 & 2 & 12 \\
\hline Cecropiaceae & 4 & 3 & 16 \\
\hline Celastraceae & 3 & 2 & 20 \\
\hline Chrysobalanaceae & 12 & 4 & 237 \\
\hline Clusiaceae & 8 & 6 & 50 \\
\hline Cochlospermaceae & 1 & 1 & 2 \\
\hline Combretaceae & 4 & 2 & 27 \\
\hline Connaraceae & 1 & 1 & 3 \\
\hline Convolvulaceae & 1 & 1 & 3 \\
\hline Dichapetalaceae & 1 & 1 & 5 \\
\hline Dilleniaceae & 1 & 1 & 2 \\
\hline Ebenaceae & 1 & 1 & 2 \\
\hline Elaeocarpaceae & 2 & 1 & 36 \\
\hline Euphorbiaceae & 17 & 11 & 117 \\
\hline Fabaceae & 21 & 10 & 94 \\
\hline Flacourtiaceae & 4 & 3 & 17 \\
\hline Hippocrateaceae & 1 & 1 & 2 \\
\hline Humiriaceae & 7 & 6 & 35 \\
\hline Lacistemaceae & 1 & 1 & 2 \\
\hline Lauraceae & 12 & 5 & 201 \\
\hline Lecythidaceae & 14 & 8 & 438 \\
\hline Malpighiaceae & 3 & 2 & 7 \\
\hline Melastomataceae & 4 & 3 & 67 \\
\hline Meliaceae & 7 & 5 & 203 \\
\hline Menispermaceae & 2 & 1 & 6 \\
\hline Mimosaceae & 15 & 6 & 218 \\
\hline Monimiaceae & 2 & 1 & 6 \\
\hline Moraceae & 19 & 12 & 229 \\
\hline
\end{tabular}

\begin{tabular}{lccc}
\hline \hline \multicolumn{1}{c}{ FAMÍLIA } & ESPÉCIES & GÊNEROS & INDIVÍDUOS \\
\hline Myristicaceae & 9 & 4 & 129 \\
Myrtaceae & 4 & 3 & 57 \\
Nyctaginaceae & 1 & 1 & 12 \\
Ochnaceae & 2 & 2 & 5 \\
Olacaceae & 2 & 2 & 15 \\
Opiliaceae & 1 & 1 & 14 \\
Quiinaceae & 4 & 4 & 6 \\
Rubiaceae & 9 & 6 & 58 \\
Rutaceae & 1 & 1 & 1 \\
Sapindaceae & 5 & 4 & 21 \\
Sapotaceae & 19 & 11 & 597 \\
Simaroubaceae & 5 & 3 & 27 \\
Sterculiaceae & 6 & 2 & 52 \\
Tiliaceae & 5 & 4 & 50 \\
Verbenaceae & 1 & 1 & 13 \\
Violaceae & 4 & 3 & 49 \\
Vochysiaceae & 3 & 2 & 16 \\
\hline TOTAL & 359 & 217 & 4583 \\
\hline \hline & & & \\
\hline
\end{tabular}

\section{Composição florística}

No levantamento realizado nas 13 unidades amostrais foram registrados 4.583 indivíduos (DAP $\geqslant 10 \mathrm{~cm}$ ) distribuídos em 55 famílias, 217 gêneros e 359 espécies, incluindo árvores, cipós e palmeiras.

A Tabela 1 mostra a distribuição geral do número de indivíduos, gêneros e espécies por família botânica com DAP $\geqslant 10 \mathrm{~cm}$. As famílias Sapotaceae, Burseraceae, Caesalpiniaceae e Lecythidaceae foram as mais representativas, com 597; 461; 440 e 438 indivíduos, respectivamente. Estas quatro famílias representam conjuntamente $42,24 \%$ de todos os indivíduos estudados. Entretanto, as famílias Rutaceae (1), Cochlospermaceae (2), Dilleniaceae (2), Ebenaceae (2), Hippocrateaceae (2), Lacistemaceae (2), Connaraceae (3) e Convolvulaceae (3) apresentaram ( 15 indivíduos), representando $0,32 \%$ do total dos indivíduos registrados.

Observa-se ainda na Tabela 1 , que as famílias representadas com maior número de gêneros são Caesalpiniaceae (20), Moraceae (12), Euphorbiaceae (11), Sapotaceae (11), Fabaceae (10), Annonaceae (9) e Apocynaceae (9), representando $37,78 \%$ do total. Entretanto, 17 famílias apresentaram somente um gênero cada, representando 30,90\%.

Em relação ao número de espécies, as famílias mais 


\section{ACTA \\ AMAZONICA}

ASPECTOS FLORÍSTICOS DE 13 HECTARES

DA ÁREA DE CACHOEIRA PORTEIRA-PA diversificadas na área de estudo foram: Caesalpiniaceae (36), Fabaceae (21), Moraceae (19), Apocynaceae (18), Euphorbiaceae (17) e Mimosaceae (15), representando $35,09 \%$ do total de espécies inventariadas. Por outro lado, foi observado que 11 famílias tiveram somente 1 espécie representando $20 \%$ da diversidade estudada.

As espécies identificadas neste trabalho, em boa parte são freqüentes na área estudada ou em todo caso, um grande número destas espécies é comum na Amazônia.
Segundo Ducke \& Black (1954) é um fato a ser considerado que na Amazônia a longitude desempenha um papel muito mais importante que a latitude na composição florística, ou seja, a diferença da flora é muito mais acentuada entre Belém e Santarém que entre Belém e Caiena. Embora no último caso, a distância seja maior, onde os dados acusam número maior de espécies para o centro e noroeste da Amazônia que para as partes orientais e ocidentais da região.

Tabela 2. Relação das 30 espécies com maior Índice de Valor de Importância - I.V.I.E. (DAP $10 \mathrm{~cm})$ na área de Cachoeira Porteira / PA.

\begin{tabular}{|c|c|c|c|c|c|}
\hline$N^{\circ}$ & ESPÉCIE & $A(\%)$ & $\mathrm{D}(\%)$ & $F(\%)$ & IVIE (\%) \\
\hline 1 & Eschweilera coriacea (DC.) S. A. Mori & 6,76 & 7,46 & 1,01 & 15,24 \\
\hline 2 & Micropholis guyanensis (A .DC.) Pierre & 5,02 & 4,84 & 1,01 & 10,87 \\
\hline 3 & Protium subserratum Engl. & 4,60 & 1,68 & 1,01 & 7,30 \\
\hline 4 & Licania hypoleuca Benth. & 2,81 & 1,93 & 1,01 & 5,76 \\
\hline 5 & Protium trifoliolata Engl. & 2,99 & 1,51 & 1,01 & 5,51 \\
\hline 6 & Swartzia laevicarpa Amsh. & 1,61 & 2,81 & 1,01 & 5,44 \\
\hline 7 & Inga marginata Willd. & 2,47 & 1,87 & 1,01 & 5,34 \\
\hline 8 & Ocotea opifera Mart. & 2,27 & 1,74 & 1,01 & 5,02 \\
\hline 9 & Tetragastris altissima (Aubl.) Swartz. & 2,09 & 1,48 & 1,01 & 4,59 \\
\hline 10 & Trichilia micrantha Benth. & 1,96 & 1,11 & 1,01 & 4,09 \\
\hline 11 & Oenocarpus bacaba Mart. & 2,38 & 0,61 & 1,01 & 4,00 \\
\hline 12 & Eschweilera fracta R. Knuth. & 1,57 & 1,52 & 0,91 & 4,00 \\
\hline 13 & Eperua glabriflora R. S. Cowan. & 1,09 & 1,68 & 0,71 & 3,48 \\
\hline 14 & Pourouma guianensis Aubl. & 1,48 & 0,91 & 0,91 & 3,30 \\
\hline 15 & Macrolobium limbatum Spruce ex Benth. & 1,31 & 1,12 & 0,81 & 3,24 \\
\hline 16 & Bocoa viridiflora (Ducke) R. S.Cowan. & 1,31 & 0,85 & 1,01 & 3,17 \\
\hline 17 & Micropholis casiquirensis Aubrév. & 1,35 & 0,91 & 0,91 & 3,17 \\
\hline 18 & Guarea carinata Ducke & 1,29 & 0,83 & 0,91 & 3,03 \\
\hline 19 & Mouriri grandiflora $D C$. & 1,24 & 0,71 & 0,96 & 2,91 \\
\hline 20 & Iryanthera ulei Warb. & 1,37 & 0,57 & 0,91 & 2,86 \\
\hline 21 & Goupia glabra Aubl. & 0,37 & 1,67 & 0,66 & 2,70 \\
\hline 22 & Chrysophyllum manaosensis Aubr. \& Pellegr. & 0,94 & 0,80 & 0,96 & 2,69 \\
\hline 23 & Osteophloeum platyspermum (A. DC.) Warb. & 0,28 & 1,77 & 0,51 & 2,56 \\
\hline 24 & Helicostylis podogyne Ducke & 0,94 & 0,78 & 0,81 & 2,52 \\
\hline 25 & Micropholis guyanensis subsp. duckeana T. D. Penn. & 1,00 & 0,66 & 0,81 & 2,48 \\
\hline 26 & Pouteria multiflora Eyma. & 0,74 & 0,82 & 0,86 & 2,42 \\
\hline 27 & Alexa sp. & 0,44 & 1,33 & 0,61 & 2,37 \\
\hline 28 & Xylopia benthamii R. E. Fr. & 0,94 & 0,60 & 0,76 & 2,29 \\
\hline 29 & Lueheopsis sp. & 0,79 & 0,72 & 0,76 & 2,27 \\
\hline 30 & Licania micrantha Miq. & 0,74 & 0,66 & 0,86 & 2,26 \\
\hline
\end{tabular}

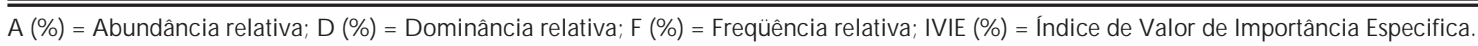




\section{ACTA \\ AMAZONICA}

ASPECTOS FLORÍSTICOS DE 13 HECTARES

DA ÁREA DE CACHOEIRA PORTEIRA-PA

\section{Estrutura horizontal}

Para analisar-se a estrutura horizontal da floresta em estudo foi necessário conhecer os parâmetros da abundância, dominância e freqüência, os quais foram obtidos através dos indivíduos com DAP $\geqslant 10 \mathrm{~cm}$.

Assim, foram registrados 4.583 indivíduos distribuídos ao longo das unidades amostrais. Os resultados mostraram que as espécies com maior abundância foram Eschweilera coriacea Mori com 310 indivíduos (6,76\%) do total; Micropholis guyanensis Pierr. 230 (5,02\%), Protium subserratum Engl. com 211 indivíduos (4,60\%), Protium trifoliolatum Engl. com 137 (2,99\%) e Licania bypoleuca Benth.com 129 (2,81\%), Tab. 2. As espécies que apresentaram os menores valores foram Paypayrola sp., Simaba guianensis Aubl., Micropholis williamii Aubr., Duroia sp. e Chimarrbis turbinata DC., todas com 1 indivíduo cada $(0,02 \%)$.

A distribuição dos indivíduos na área de estudo mostrou que as espécies diferem uma das outras quanto a sua área basal. Com isso, as espécies que mais se destacaram foram Eschweilera coriacea Mori. (7,46\%), Micropbolis guyanensis Pierr.. (4,84\%), Swartzia laevicarpa Amsh. (2,81\%0, Licania bypoleuca Benth. (1,93\%) e Inga marginata Willd. (1,87\%), Tab. 2. As espécies que apresentaram menores valores foram Quiina sp., Touroulia sp., Duroia sp., Sapium marmiere Hub. e Paypayrola sp., todas com $0,08 \%$.

As espécies com maior distribuição nas parcelas estudadas e maiores valores de freqüência foram Oenocarpus bacaba Mart., Protium subserratum Engl. Tetragastris altíssima Aubl. e Bocoa viridiflora Ducke, todas com 1,01\%, (Tab. 2). Por outro lado, as espécies com os menores valores foram Paypayrola sp., Simaba guianensis Aubl., Micropholis williamii Aubr., M. mensalis Baehn. e Duroia sp., todas com 0,05\%.

De posse dos dados de abundância, dominância e freqüência relativa, foram determinadas as espécies que alcançaram os maiores valores do IVIE, as quais foram Eschweilera coriacea (DC.) Mori com 15,24; Micropbolis guyanensis Pierr. 10,87 e Protium subserratum Engl. com 7,30; representando 5,08\%; 3,62\% e 2,43\% respectivamente do total (Tab. 2). As espécies que apresentaram valores menores foram Astrocaryum aculeatum Meyer e Duroia sp. com 0,07, representando 0,023\% em toda área.

A Tabela 2 mostra ainda, que os parâmetros abundância, dominância e freqüência variam muito em relação às espécies, e isto poderia ser explicado pela variação acentuada dos relevos, tipo de solo e drenagem. Por outro lado, há uma discrepância muito alta entre o percentual de densidades das árvores e a sua área basal. A resposta para isso está no diâmetro dos indivíduos, onde algumas espécies apresentaram um grande número de indivíduos com diâmetros pequenos, entretanto outras espécies apresentaram poucos indivíduos, mas possuíam grandes diâmetros, como, por exemplo, as "melancieiras" (Alexa grandiflora Ducke).

Com relação à diversidade relativa (razão entre o número de espécies da família pelo total de espécies) esta mostra que as famílias Caesalpiniaceae com 10,09\% representa o maior percentual, seguida de Moraceae com 6,5\%; Fabacaeae com 5,76\%; Sapotaceae com 5,48\% e Mimosaceae com 4,61\% (Tab.3). As famílias com menor diversidade relativa foram Rutaceae com $0,32 \%$; Lacistemaceae e Hippocrateaceae com 0,34\%; Ebenaceae e Dilleniaceae com $0,35 \%$ cada uma.

A densidade relativa ( razão entre o número de indivíduos da família pelo total de famílias) mostra que as famílias com maiores valores foram Sapotaceae com 13,03\%; Burseraceae 10,06\%; Caesalpiniaceae 9,60\%; Lecythidaceae 9,56\% e Chrysobalanaceae com 5,17\% (Tab.3). Por outro lado, as famílias Rutaceae com 0,02\%; Lacistemaceae; Hippocrateaceae; Ebenaceae e Dilleniaceae todas com $0,04 \%$ obtiveram os menores valores de diversidade relativa.

A dominância relativa (razão entre a área basal da família pela área basal total) apresentou as famílias Sapotaceae com 11,83\%; Caesalpiniaceae 11,76\%; Lecythidaceae 11,54\%; Lauraceae 6,58\% e Fabaceae com 6,07\% como destaques (Tab.3).Todavia, as famílias Rutaceae, Lacistemaceae, Hippocrateaceae e Ebenaceae com 0,01\% e Dilleniaceae com $0,02 \%$ apresentaram os menores valores de dominância.

Em relação ao Índice de Valor de Importância das Famílias (IVIF), as famílias com maiores valores foram Caesalpiniaceae com 31,45 (10,48\%), seguidas das Sapotaceae com 30,33, (10,11\%), Lecythidaceae 24,27, $(8,09 \%)$, Burseraceae 16,99 (5,66\%) e Moraceae 16,13, $(5,37 \%)$, (Tab.3). As famílias com menores valores do IVIF foram Hippocrateaceae e Lacistemaceae com $0,34(0,11 \%)$ e Rutaceae com $0,32(0,10 \%)$.

Assim, observou-se que a família Leguminosae composta por Caesalpiniaceae, Fabaceae e Mimosaceae alcançou o maior valor do IVIF na estrutura das vegetações estudadas com 59,41, representando em percentagens $19,80 \%$ em toda área estudada.

Estes resultados só comprovam Ducke e Black (1954), os quais afirmaram que a família de maior importância dentro da floresta amazônica é sem dúvida a das Leguminosae, e que essa família, também é responsável depois das palmeiras, pelo elemento fisionômico da flora.

\section{CONCLUSÃO}

A vegetação encontrada na área de estudo em OriximináPA é coberta por uma mata natural sem muita interferência antrópica. A região apresenta muitos relevos diversificados, e com isso, há uma heterogeneidade de espécies dentro de cada comunidade vegetal, sendo a mais marcante a vegetação na terra firme, onde, segundo a fotointerpretação, ocorre uma diversidade local acentuada o que foi constatado 


\section{ACTA \\ AMAZONICA}

ASPECTOS FLORÍSTICOS DE 13 HECTARES

DA ÁREA DE CACHOEIRA PORTEIRA-PA no campo. As demais comunidades ocorrem em menor extensão, mas com um grande valor ecológico, isto devido as mesmas apresentarem uma quantidade bem elevada de endemismos de espécies, como, por exemplo, a vegetação que ocorre nas comunidades das cachoeiras.

Os dados do levantamento florístico mostraram a mudança do relevo, drenagem e tipo de solo, que culminam com os agrupamentos em manchas heterogêneas, com dominância de algumas espécies, e sempre melhoradas por outras, geralmente de 20 a 25 novas espécies, a cada unidade amostrada.

Verificou-se que as 30 espécies com maior Índice de Valor de Importância (IVI), representam em torno aproximadamente de $54 \%$ do perfil estrutural da floresta estudada, enquanto as famílias representam em torno de $90 \%$ aproximadamente.

Tabela 3. Relação das 30 famílias com maior Índice de Valor de Importância Familiar - I.VII.F. (DAP $\geqslant 10 \mathrm{~cm})$ na área de Cachoeira Porteira / PA

\begin{tabular}{|c|c|c|c|c|c|}
\hline $\mathrm{N}^{0}$ & FAMÍLIA & $\overline{~ D I V ~(\%) ~}$ & $\overline{\text { DEN (\%) }}$ & DOM (\%) & I.V.I F (\%) \\
\hline 1 & Caesalpiniaceae & 10,09 & 9,60 & 11,76 & 31,45 \\
\hline 2 & Sapotaceae & 5,48 & 13,03 & 11,83 & 30,34 \\
\hline 3 & Lecythidaceae & 3,17 & 9,56 & 11,54 & 24,27 \\
\hline 4 & Burseraceae & 2,02 & 10,06 & 4,91 & 16,99 \\
\hline 5 & Moraceae & 6,05 & 5,00 & 5,09 & 16,14 \\
\hline 6 & Lauraceae & 3,75 & 4,39 & 6,58 & 14,72 \\
\hline 7 & Mimosaceae & 4,61 & 4,76 & 4,70 & 14,07 \\
\hline 8 & Fabaceae & 5,76 & 2,05 & 6,07 & 13,88 \\
\hline 9 & Chrysobalanaceae & 3,17 & 5,17 & 3,81 & 12,15 \\
\hline 10 & Meliaceae & 2,02 & 4,43 & 3,11 & 9,56 \\
\hline 11 & Annonaceae & 3,75 & 3,36 & 1,79 & 8,90 \\
\hline 12 & Euphorbiaceae & 4,32 & 2,55 & 1,54 & 8,41 \\
\hline 13 & Myristicaceae & 2,31 & 2,81 & 3,23 & 8,35 \\
\hline 14 & Apocynaceae & 4,90 & 1,53 & 1,59 & 8,02 \\
\hline 15 & Arecaceae & 2,59 & 3,60 & 0,96 & 7,15 \\
\hline 16 & Combretaceae & 1,15 & 0,59 & 4,06 & 5,80 \\
\hline 17 & Anacardiaceae & 2,59 & 1,20 & 1,42 & 5,21 \\
\hline 18 & Rubiaceae & 2,59 & 1,27 & 0,88 & 4,74 \\
\hline 19 & Humiriaceae & 2,02 & 0,76 & 1,15 & 3,93 \\
\hline 20 & Clusiaceae & 2,31 & 1,09 & 0,50 & 3,90 \\
\hline 21 & Caryocaraceae & 0,86 & 0,26 & 2,56 & 3,68 \\
\hline 22 & Sterculiaceae & 2,02 & 1,13 & 0,30 & 3,45 \\
\hline 23 & Melastomataceae & 1,15 & 1,46 & 0,79 & 3,40 \\
\hline 24 & Tiliaceae & 1,15 & 1,09 & 1,14 & 3,38 \\
\hline 25 & Bombacaceae & 2,02 & 0,44 & 0,57 & 3,03 \\
\hline 26 & Celastraceae & 0,58 & 0,44 & 1,88 & 2,90 \\
\hline 27 & Myrtaceae & 1,15 & 1,24 & 0,49 & 2,88 \\
\hline 28 & Bignoniaceae & 1,15 & 0,39 & 1,10 & 2,64 \\
\hline 29 & Simaroubaceae & 1,15 & 0,59 & 0,86 & 2,60 \\
\hline 30 & Violaceae & 1,15 & 1,07 & 0,25 & 2,47 \\
\hline
\end{tabular}

DIV $(\%)$ = Diversidade relativa; DEN (\%) = Densidade relativa; DOM (\%) = Dominância relativa; IVIF (\%) Índice de Valor de Importância das Famílias. 


\section{ACTA AMAZONICA}

\section{AGRADECIMENTOS}

Os autores agradecem ao técnico José Lima dos Santos da Coordenação de Pesquisas em Botânica do INPA, pela valiosa ajuda nas identificações do material botânico.

\section{BIBLIOGRAFIA CITADA}

Alencar, J. da C.1986. Análise da Associação e estrutura de uma comunidade de Floresta Tropical Úmida, onde ocorre, Aniba roseadora Ducke(Lauraceae). Tese de Doutorado. Manaus. INPA/FUA.206 p.

Cain, S. A.; Castro, G. M. de ; Pires, J. M.; Silva, N. T. 1956. Application of some phytosociological techniques to Brazilian rain forest. American Journal of Botany, 43 (10): 911-941.

Cain, S. A.; Castro, G. M. de. 1959. Manual of vegetation analysis. Hafner Publishing Company. New.York, USA.325 p.

Conceição, M. C. A . 1990. Análise estrutural de uma floresta de várzea no estado do Pará. Dissertação de Mestrado, UPFR. Curitiba, Paraná. 170 p.

Cronquist, A. 1981. An Integrated System of Classification of Flowering Plants. Columbia Univ. Press, New York, USA. 1261 p.

Ducke, A.; Black, G. A. 1954. Notas sobre a fitogeografia da Amazônia Brasileira. Bol. Téc. do Inst. Agron. Norte, Belém, (29): 1-48.

Finol, U. H. 1971. Nuevos parametros a considerarse em el análisis estructural de las selvas virgines tropicales. Ver. For. Venez., 14(21): 29-42.

Förster, M. 1973. Strukturanalyses eines tropischen Regenwaldes in Kolumbien. Allg. Forst. v. J. ZTG., 19 (26): 17-44.

Hosokawa, R. T. 1981. Manejo de florestas tropicais úmidas em regime de rendimento sustentado. Relatório técnico, UFPR. Curitiba, Paraná. 125 p.

Jardim, F. C. 1985. Estrutura de floresta equatorial úmida da estação experimental de silvicultura tropical do INPA. Dissertação de mestrado. Manaus. INPA/FUA. 195 p.

Lamprecht, H. 1962. Ensayo sobre unos metodos para el analisis estructural de los bosques tropicales. Acta Cientifica Venezolana, 13 (2): 57-65.

Lamprecht, H. 1964. Ensayo sobre la estructura floristica del parte sur-oriental del bosque universitário " El Caimital " Estado Baridas. Ver. For. Venez., 7 (10-11): 77-119.

Lima Filho, D. A .; Matos, F. D. A.; Amaral, I. L; Revilla, J.; Coelho, L. S; Ramos, J. F.; Santos, J. L. 2001. Inventário florístico de floresta ombrófila densa de terra firme, na região do rio Urucu Amazonas, Brasil. Acta Amazonica, 31(4): 565-579.
Lisboa, P.L. 1975. Observações gerais e revisão bibliográfica sobre as campinas amazônicas de areia branca. Acta Amazonica, (3): $211-223$.

Longhi, S. V. 1980. A estrutura de uma floresta natural de Araucaria angustifolia (Bert.) O. Ktze, sul do Brasil. Dissertação de Mestrado, UFPR. Curitiba, Paraná. 198 p.

Matos, F. D. A ; Amaral, I. L. 1999. Análise ecológica de um hectare em floresta ombrófila densa de terra-firme, estrada da Várzea, Amazonas, Brasil. Acta Amazonica, 29(3): 365-379.

Montoya Maquin, J.M; Matos, G.F. 1967. Um enfoque fisionômico - estrutural para la descripcion de la vegetacion. Turrialba, 17 (2): 197 - 207.

Mori, A.S.; Boom, B.N.; Carvalho, A.M.; Santos, T.S. 1983. Ecological importance of Myrtaceae in an Eastern Brazilian wet forest. Biotropica, 15 (1): 68 - 70.

Oliveira, A. A. 2000. Inventários quantitativos de árvores em floresta de terra firme: Revisão com enfoque na Amazônia Brasileira. Acta Amazonica, 30(4): 543-567.

Pires, J.M. 1973. Tipos de vegetação da Amazônia. Publicação Avulsa Museu Paraense Emílio Goeldi. Belém, 20: 179 - 202.

Revilla, J.; Lleras, E.; Rodrigues, W. A .1981. Levantamento, identificação e cadastramento da flora de Tucuruí. Relatório técnico, Convênio INPA/ELN. Manaus-AM. 266p.

Revilla, J.; Lima Filho, D.A.; Amaral, I. L.; Matos, F.D.A. 1986. Estudos e levantamentos do impacto ambiental da futura UHE de Cachoeira Porteira - PA. Relatório, ENGE-RIO/INPA. 73p.

Rizzini, C.T. 1963. Nota prévia sobre a divisão fitogeográfica (florístico - sociológica) do Brasil. Revista Brasileira de Geografia, 25 (1) : 3 - 64.

Rodrigues, W.A. 1961. Estudo preliminar da mata de várzea alta de uma linha do baixo Rio Negro, de solo argiloso e úmido. INPA Publ. Botânica, 10: 1 - 28.

Souza, P.F. 1973. Terminologia florestal glossário de termos e expressões florestais. Rio de Janeiro, Fundação IBGE, 304 p.

Veiga, A. de A. 1977. Glossário em Dasonomia. São Paulo, Instituto Florestal, 97 p.

\section{RECEBIDO EM 26/03/2003 \\ ACEITO EM 17/07/2004}

